\title{
A novel multiple-marker method for the early diagnosis of oral squamous cell carcinoma
}

\author{
Jutta Ries ${ }^{\mathrm{a}, *}$, Nur Mollaoglu ${ }^{\mathrm{a}, \mathrm{b}}$, Takeshi Toyoshima $^{\mathrm{c}}$, Eleftherios Vairaktaris $^{\mathrm{d}}$, Friedrich W. Neukam ${ }^{\mathrm{a}}$, \\ Sabine Ponader ${ }^{\mathrm{a}}$ and Emeka Nkenke ${ }^{\mathrm{a}}$ \\ ${ }^{a}$ Department of Oral and Maxillofacial Surgery, University of Erlangen, Glückstrasse 11, 91054 Erlangen, \\ Germany \\ ${ }^{\mathrm{b}}$ Department of Oral and Maxillofacial Surgery, University of Gazi, School of Dentistry Ankara, Turkey, Emek \\ 8.cadde, 82.sokak, 06510, Ankara, Turkey \\ ${ }^{\mathrm{c}}$ Department of Oral and Maxillofacial Surgery, Kyushu University, 3-1-1 Maidashi, Higashi-ku, Fukuoka \\ 812-8582, Japan \\ ${ }^{\mathrm{d}}$ Department of Oral and Maxillofacial Surgery, University of Athens, Athens, Greece
}

\begin{abstract}
Objective: Melanoma associated antigens-A (MAGE-A) expression is highly specific to cancer cells. Thus, they can be the most suitable targets for the diagnosis of malignancy. The aim of this study was to evaluate the sensitivity of multiple MAGE-A expression analysis for the diagnosis of oral squamous cell carcinoma (OSCC).

Methods: Total of 70 OSSC and 20 normal oral mucosal (NOM) samples of otherwise healthy volunteers were examined for the expression of 10 different single antigens out of 12 different MAGE-A subtypes by highly sensitive reverse transcriptase polymerase chain reaction (RT-PCR) methods. The results were correlated to clinicopathological parameters of tumor samples. Results: Expression of MAGE-A was restricted to OSCC. The expression frequency of single antigen was between $10 \%$ and $55 \%$. However, expression rate was increased up to $93 \%$ by the elevated number of genes examined. A significant correlation was found between the expression of MAGE-A and malignancy $(p=0.0001)$. In addition, multiple MAGE-A detection has also correlated to the incidence of lymph node metastasis, grading and advanced clinical stages.

Conclusions: Analysis of multiple MAGE-A expression is more sensitive than the analysis of a single MAGE-A for the diagnostic evaluation of OSCC. Multiple MAGE-A expression analysis may be a very sensitive method to be used for the diagnosis even in the early stage of OSCC.
\end{abstract}

Keywords: Oral squamous cell carcinoma, MAGE-A expression, multiple markers, RT-PCR, diagnosis

\section{Introduction}

Oral squamous cell carcinoma (OSCC) is the 6th most frequent occurring cancer world wide [1]. Although, early stage of OSCC is often curable, the prognosis of advanced clinical stages generally remains poor. Despite the extensive researches on treatment modalities for OSCC five year survival rate has been

* Corresponding author: Dr. Jutta Ries, PhD, Department of Oral and Maxillofacial Surgery, University of Erlangen, Glückstrasse 11, 91054 Erlangen, Germany. Tel.: +49 913185 6810; Fax: +49 9131 85 4219; E-mail: Jutta.Ries@uk-erlangen.de. reported to be approximately $50 \%$ and has not been improved over the last four decades [2,3]. The major contributing factors for un-improvement in the survival rate might be the late diagnosis of primary tumors and the high incidence of local recurrences due to occurrence of occult cancer cells in tumor margins [4,5]. Furthermore, OSCC is known to exhibit "field cancerization" which may result in a second primary tumor [6]. Additionally, early dissemination of tumor cells into bone marrow or peripheral blood during the surgery may also cause recurrence and metastasis, which is usually overlooked. To increase the survival rate of patients with OSCC, highly sensitive and specific molecular diagnos- 
tic techniques to discriminate malignant from benign lesions and to detect residual or disseminated tumor cells (DTC) using multiple molecular tumor markers might be effective.

Melanoma associated antigens-A (MAGE-A) are suitable markers to be used in the early molecular diagnosis of cancer. The MAGE-A family consists of 12 subtypes including MAGE-A1 to -A12 [7-10]. Based on the reverse transcriptase polymerase chain reaction (RT-PCR) typing results for MAGE-A genes, except MAGE-A7 which is not found to be not transcribed, MAGE-A is expressed almost exclusively in various tumor types and testis [9-19]. Expression of single MAGE-A genes has already been extensively examined for the diagnostic application of different malignancies. However, tumor detection by a single antigen was not sensitive enough for the diagnostic application due to its low expression frequency and heterogeneous expression pattern, although they have a high specificity to cancer cells. Nevertheless, expression frequency was higher when multiple MAGE-A was applied. Previous studies reported that multiple MAGE-A analysis does increase the sensitivity of tumor cell detection in solid tumors including OSCC compared to single MAGE-A analysis [15-17,20-24].

There are several reports regarding to establishment of molecular diagnostic techniques for the early diagnosis of primary tumors, and also detection of rare occult tumor cells or DTC's using multiple molecular markers with definitive accuracy to malignancies including the one based on MAGE-A expression pattern [15,23-28]. Therefore, in order to increase the sensitivity of the previously established methods, it was aimed to investigate the expression rate of mRNA transcripts of 10 different MAGE-A subtypes using RT-PCR in a large number of OSCC samples to determine whether multiple expression analysis of MAGE-A is a more sensitive application for the detection of OSCC.

\section{Patients and methods}

\subsection{Patients and sample collection}

Total of 70 tumor samples obtained from patients with OSCC and 20 normal oral mucosa (NOM) samples were included to this study. All NOM tissues were obtained from retromolar area of otherwise healthy volunteers during prophylactic lower third molar removal. Sampling areas for NOM were all free of inflammation and infection. This side also was the most resembling area where all tumor samples were collected. Patients' informed consent and the approval of the local ethical committee was taken. Prior to biopsy or tumor resection, tumor patients received neither radiotherapy nor chemotherapy. Each sample taken was divided into two pieces. One piece was histologically examined and the second piece was immediately snap frozen and stored at $-80^{\circ} \mathrm{C}$ until the molecular examination. For RT-PCR analysis, RNA of normal colon and testis has been used as negative and positive controls respectively.

\subsection{Histopathology and staging}

Clinical staging and TNM classification were done for each tumor patient according to UICC [29]. All biopsies were evaluated by the same pathologist to ensure the consistent results. The OSCC were also classified according to WHO [30] for loss of differentiation as G1, G2 and G3 for the well, moderately and poorly differentiation respectively. Additional clinical information including age, gender and clinical staging (stage I to IV) were also recorded. In addition, tumor samples were classified according to early (including stage I and II) and late (including stage III and IV) clinical stages and also into two groups as $\mathrm{T} 1 \& \mathrm{~T} 2$ and T3 \& T4 displaying the early and advanced tumor staging. Moreover, state of lymph nodes was recorded as $\mathrm{N}=0$ and $\mathrm{N}>0$ for negative and positive lymph node existence respectively.

\subsection{Detection of MAGE-A expression by RT-PCR}

Total RNA from frozen tissues was isolated using RNeasy Mini Kit according to the manufacturer's instructions (Qiagen, Hilden). Total of 200 ng RNA was used for the detection of MAGE-A expression. All primers used for amplification steps are summarized in Table 1. High sensitive RT-nested PCR methods (nPCR) were used for the detection of MAGE-A1-A6 (MAGE-A1-6 Assay) and A12 (RT-nPCR) expressing tissues $[15,24,25]$.

MAGE-A1-6 Assay: Reverse transcription and the first PCR reaction were carried out using One Step RT-PCR Kit (Qiagen, Hilden, Germany) and multiple MAGE-A recognizing primers (MMRP1/2). These primers can bind to the sequences of MAGE-A1, A2, A3, A4, A5 and A-6 together and allow a simultaneous detection of all subtypes by the same method as described previously $[15,23]$. For nPCR, $1 \mu$ l of first PCR product was used as a template for the second PCR. For detection of simultaneous gene expression of MAGE- 
Table 1

Primer for amplification of GAPDH and MAGE-A members. $\mathrm{S}=$ sense; as = antisense; $\mathrm{MMRP}=$ Multiple MAGE-A 1 to A6 recognizing primers; W = A or T; M1- M6 = specific primers for MAGE-A1 to A6; o = outer primer of nested RT-PCR; I = inner primer of nested RT-PCR; *detection of two transcript variants due to different splicing

\begin{tabular}{|c|c|c|c|c|}
\hline Primer & Primer sequence & Cycles & $\begin{array}{l}\text { Size } \\
\text { (bp) }\end{array}$ & $\begin{array}{c}\text { Annealing } \\
\left({ }^{\circ} \mathrm{C}\right)\end{array}$ \\
\hline GAPDH s & GCAGGGGGGAGCCAAAAGGG & 35 & 567 & 60 \\
\hline GAPDH as & TGCCAGCCCCAGCGTCAAAG & & & \\
\hline MAGE-A1-6 Assay & & & & \\
\hline MMRP1 & CTGAAGGAGAAGATCTGCC & 30 & 831 to 855 & 60 \\
\hline MMRP2 & CTCCAGGTAGTTTTCCTGCAC & & & \\
\hline MMRP3 & CTGAAGGAGAAGATCTGCCWGTG & 30 & 496 to 493 & 60 \\
\hline MMRP4 & 5-CCAGCATTTCTGCCTTTGTGA-3 & & & \\
\hline M1 & CGGAACAAGGACTCCAGGATACAA & 30 & 375 & 60 \\
\hline M2 & GAAAGAAGTCCTGGCAATTTCTGAG & 30 & 522 & 60 \\
\hline M3 & CCAAAGACCAGCTGCAAGGAACT & 30 & 568 & 60 \\
\hline M4 & CGTCAATGCCAAAGATCATCTTCAG & 30 & 577 & 60 \\
\hline M5 & CCTTTGTGACCAGCTCCTTGACTTA & 30 & 510 & 60 \\
\hline M6 & CCAGGCAGGTGGCAAAGATGTACAC & 30 & 628 & 60 \\
\hline \multicolumn{5}{|l|}{ MAGE-A8 } \\
\hline A8 s & GGAGGCAAGGTTCGCAGAGA & 40 & 299 & 60 \\
\hline A8 as & GGAGGACTTGGTGACCCAGA & & & \\
\hline \multicolumn{5}{|l|}{ MAGE-A9 } \\
\hline A9 $\mathrm{s}$ & GACCTTGAAGCCCAAGGAGA & 35 & 300 & 58 \\
\hline A9 as & GACCTTGAAGCCCAAGGAGA & & & \\
\hline \multicolumn{5}{|l|}{ MAGE-A10* } \\
\hline A10 s & GGAACCCCTCTTTTCTACAGAC & 40 & 411 & 60 \\
\hline & & & 501 & \\
\hline A10 as & TCCTCTGGGGTGCTTGGTATTA & & & \\
\hline \multicolumn{5}{|l|}{ RT-nPCR } \\
\hline MAGE-A12os & TCCGTGAGGAGGCAAGGTTC & 30 & 341 & 60 \\
\hline MAGEA12oas & ATCGGATTGACTCCAGAGAGTA & & & \\
\hline MAGE-A12is & TCCGTGAGGAGGCAAGGTTC & 30 & 181 & 58 \\
\hline MAGEA12ias & GAGCCTGCGCACCCACCAA & & & \\
\hline
\end{tabular}

A1 to A6, a primer set recognizing MAGE-A was applied (MMRP3 and MMRP4). To assign the individual MAGE isotype, a combination of MMRP3 and one of MAGE isotype specific primers M1 to M6 were used. PCR products were separated on $1 \%$ agarose gels and stained with ethidium bromide in order to identify the different amplification products by size (Fig. 1).

RT-nPCR for MAGE-A12: Reverse transcription and the first PCR reaction was carried out using One Step RT-PCR Kit (Qiagen, Hilden) and outer MAGEA12 specific primers that were previously described. $1 \mu \mathrm{l}$ of the first PCR amplification was applied to the second PCR using inner MAGE-A12 primers (Table 1) $[25,31]$. Polymerase PCR products were separated on a $1.5 \%$ agarose gel and stained with ethidium bromide. The size of the fragment amplificated by RT-PCR was 181 bp (Fig. 1).

For the other members of MAGE-A, only one amplification step was performed using One Step RT-PCR Kit (Qiagen, Hilden, Germany). Primer sets, number of cycles, sizes of amplification products as well as annealing temperature have been summarized in Table 1. Cycling conditions for all RT-PCR steps were equal for all reactions: Reverse transcription was done at $50^{\circ} \mathrm{C}$ for 30 minutes; initial PCR activation step was performed at $95^{\circ} \mathrm{C}$ for 5 minutes; denaturation happened at $95^{\circ} \mathrm{C}$ for 30 seconds; elongation was done at $72^{\circ} \mathrm{C}$ for 45 seconds with a final extension incubation at $72^{\circ} \mathrm{C}$ for 10 minutes.

To exclude false negative results, quality of RNA was assessed by One Step RT-PCR Kit amplifying cDNA using glyceraldehyde-3-phosphate-dehydrogenase (GAPDH)-specific primers for the reaction and analysing the GAPDH-PCR products by a $2 \%$ agarose gel (Fig. 1). To exclude false positive results generated by amplification of genomic DNA sequences that were not totally eliminated by DNAs digestion, $1 \mu \mathrm{l}$ of purified RNAs from each specimen were tested for amplification of genomic GAPDH using the specific primers for PCR. Only RNA isolations showing no visible band in 
a

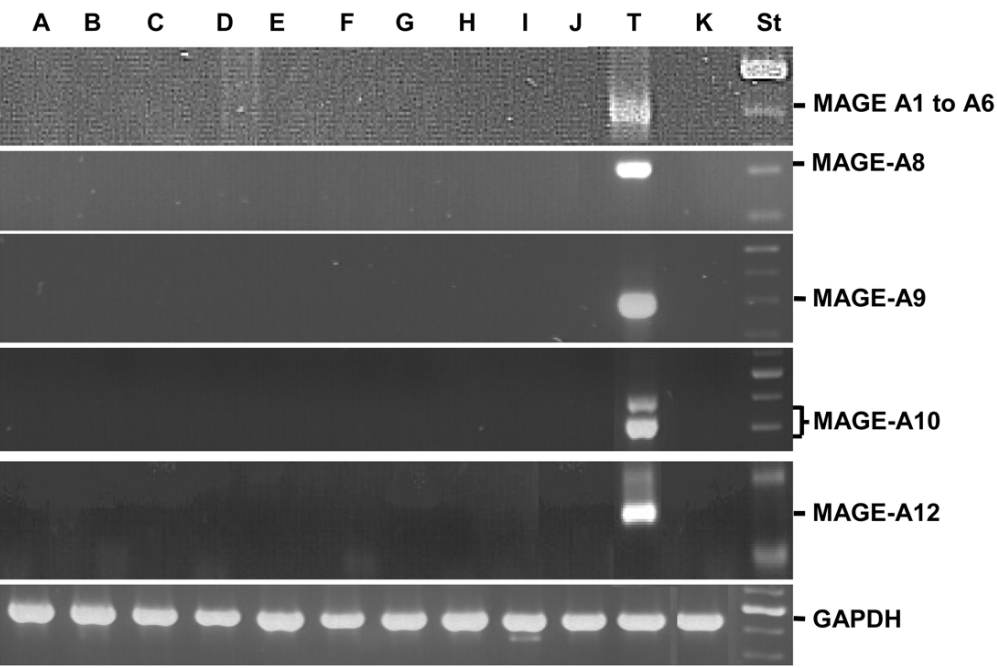

b
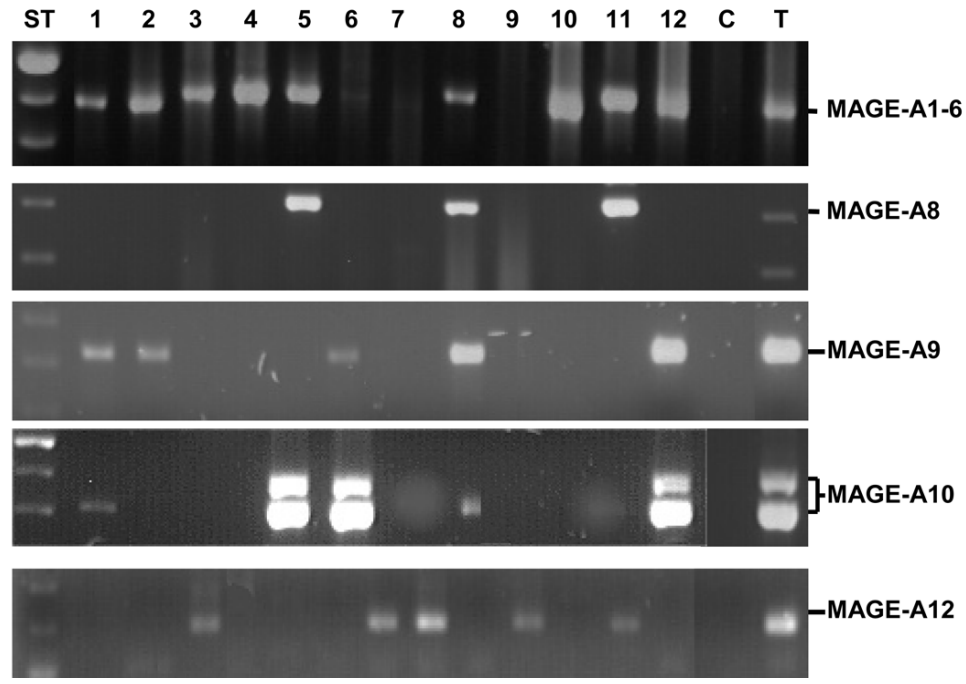

Fig. 1. Expression analysis of MAGE-A in normal oral mucosa (NOM) and OSCC. $\mathrm{T}=\mathrm{Testis}$; $\mathrm{C}=\mathrm{Colon}$; ST $=$ Standard. A) Analysis of MAGE-A expression in NOM (A-J). The quality of RNA was assessed amplifying cDNA using glyceraldehyde-3-phosphate-dehydrogenase (GAPDH) specific primers for PCR. The expression of MAGE-A was not found in NOM. B) Simultaneous detection of MAGE-A1 to A6, -A8, $-\mathrm{A} 9,-\mathrm{A} 10$ and $-\mathrm{A} 12$ in samples of OSCC (1-12) are displayed respectively

$2 \%$ agarose gel were analyzed for the subsequent procedures. Each RT-PCR experiment was performed in triplicate using the same RNA sample and appropriate controls.

\subsection{Statistical analysis}

Statistical analysis was performed using the statistical software package of SPSS 16 (Inc., Chicago, USA). Association between detection of at least one of the MAGE-A and clinicopathological parameters were analysed by $\chi^{2}$ test. The prevalence of MAGE-A expression in OSCC and NOM was also compared by $\chi^{2}$ test, and $\mathrm{p}$ value less than 0.05 was considered as an indication of a statistical significance.

\section{Results}

Fifty-seven of 70 tumor patients were male and 13 of them were female. The mean age was 58.5 years (Min: 34 and Max: 91). Regarding to tumor size 21 patients were at stage $\mathrm{T} 1,13$ at T2, 5 at T3, 27 at T4. In addition, there were 31 patients with no incidence of lymph node metastasis. Moreover, 18 patients were clinically at stage I, nine at stage II, seven at stage III, and 32 were 
Table 2

Association between the expression of at least one gene out of nine MAGE-A applied and the clinicopathological parameters of patients suffer from OSCC

\begin{tabular}{|c|c|c|c|c|c|}
\hline & No. of cases & $(+)$ & $(-)$ & Positive \% & $\mathrm{P}$ value \\
\hline \multicolumn{6}{|l|}{ Diagnosis } \\
\hline SCC & 70 & 65 & 5 & 93 & 0.0001 \\
\hline NOM & 20 & 0 & 20 & 0 & \\
\hline \multicolumn{6}{|l|}{ Differentiation degree } \\
\hline No. of valid cases* & 67 & 62 & 5 & 92.5 & \\
\hline G1 & 3 & 2 & 1 & 66.7 & 0.031 \\
\hline $\mathrm{G} 2$ & 46 & 45 & 1 & 97.8 & \\
\hline G3 & 18 & 15 & 3 & 83.3 & \\
\hline \multicolumn{6}{|l|}{ Tumor size } \\
\hline No. of valid cases* & 66 & 61 & 5 & 92.4 & \\
\hline 1 & 21 & 17 & 4 & 81 & 0.87 \\
\hline 2 & 13 & 12 & 1 & 92.3 & \\
\hline 3 & 5 & 5 & 0 & 100 & \\
\hline 4 & 27 & 27 & 0 & 100 & \\
\hline \multicolumn{6}{|l|}{ grouped } \\
\hline $\mathrm{T} 1 \& \mathrm{~T} 2$ & 34 & 29 & 5 & 85.3 & 0.024 \\
\hline $\mathrm{T} 3 \& \mathrm{~T} 4$ & 32 & 32 & 0 & 100 & \\
\hline \multicolumn{6}{|c|}{ State of lymph node $(\mathrm{N})$} \\
\hline No. of valid cases* & 64 & 60 & 4 & 93.8 & \\
\hline $\mathrm{Nx} *$ & 3 & 3 & 0 & 100 & 0.126 \\
\hline 0 & 31 & 27 & 4 & 87.1 & \\
\hline 1 & 10 & 10 & 0 & 100 & \\
\hline $2 * *$ & 20 & 20 & 0 & 100 & \\
\hline N 0 & 31 & 27 & 4 & 87.1 & 0.042 \\
\hline $\mathrm{N} 1+2$ & 30 & 30 & 0 & 100 & \\
\hline \multicolumn{6}{|l|}{ Stage } \\
\hline No. of valid cases* & 66 & 61 & 5 & 92.4 & \\
\hline Early (I, II) & 27 & 22 & 5 & 81.5 & 0.005 \\
\hline Late (III, IV) & 39 & 39 & 0 & 100 & \\
\hline I & 18 & 15 & 3 & 83.3 & 0.044 \\
\hline II & 9 & 7 & 2 & 77.8 & \\
\hline III & 7 & 7 & 0 & 100 & \\
\hline IV & 32 & 32 & 0 & 100 & \\
\hline
\end{tabular}

Valid cases*: number of cases for which the clinico-pathological characteristics $(\mathrm{G}, \mathrm{T}, \mathrm{N})$ were determined. Cases without these determination were excluded from statistical analysis.

$\mathrm{Nx} * *$ Lymph node could not be classified; $2 * * *$ covers stages $2 \mathrm{a}, 2 \mathrm{~b}$ and $2 \mathrm{c}$.

at stage IV. Among all the cases three were well differentiated OSCC, 46 were moderately differentiated, and 18 were poorly differentiated (Table 2 ).

\subsection{Expression of a single MAGE-A in OSCC and NOM}

Expression of MAGE-A was restricted to OSCC (Fig. 1). None of them was expressed in NOM (Fig. 1a). However, expression frequencies of each gene varied largely in tumor tissues (Fig. 1b; Fig. 2a). Additionally, the number and type of expressed genes varied. No common occurrence of a single MAGE-A has been detected between the different individual subtypes (Fig. 1b).
The highest expressed antigen was MAGE-A4 (55\%) followed by MAGE-A9 (50\%) and A12 (50\%). The rest was as follows A3 (46\%), A6 (44\%), A10 (41\%), A1 (36\%), A2 (28\%) and A5 (25\%). Only $10 \%$ of the all OSCC cases expressed MAGE-A8 (Fig. 2a). These expression ratios were obtained when the genes were applied as a single gene.

\subsection{Expression frequencies in OSCC by multiple $M A G E-A$ analysis}

Among all OSCC samples, 93\% expressed at least one antigen when all ten of the MAGE-A genes were applied as multiple gene markers. In the early clinical stages (Stage I and II) $81 \%$ of the tumor cases expressed 

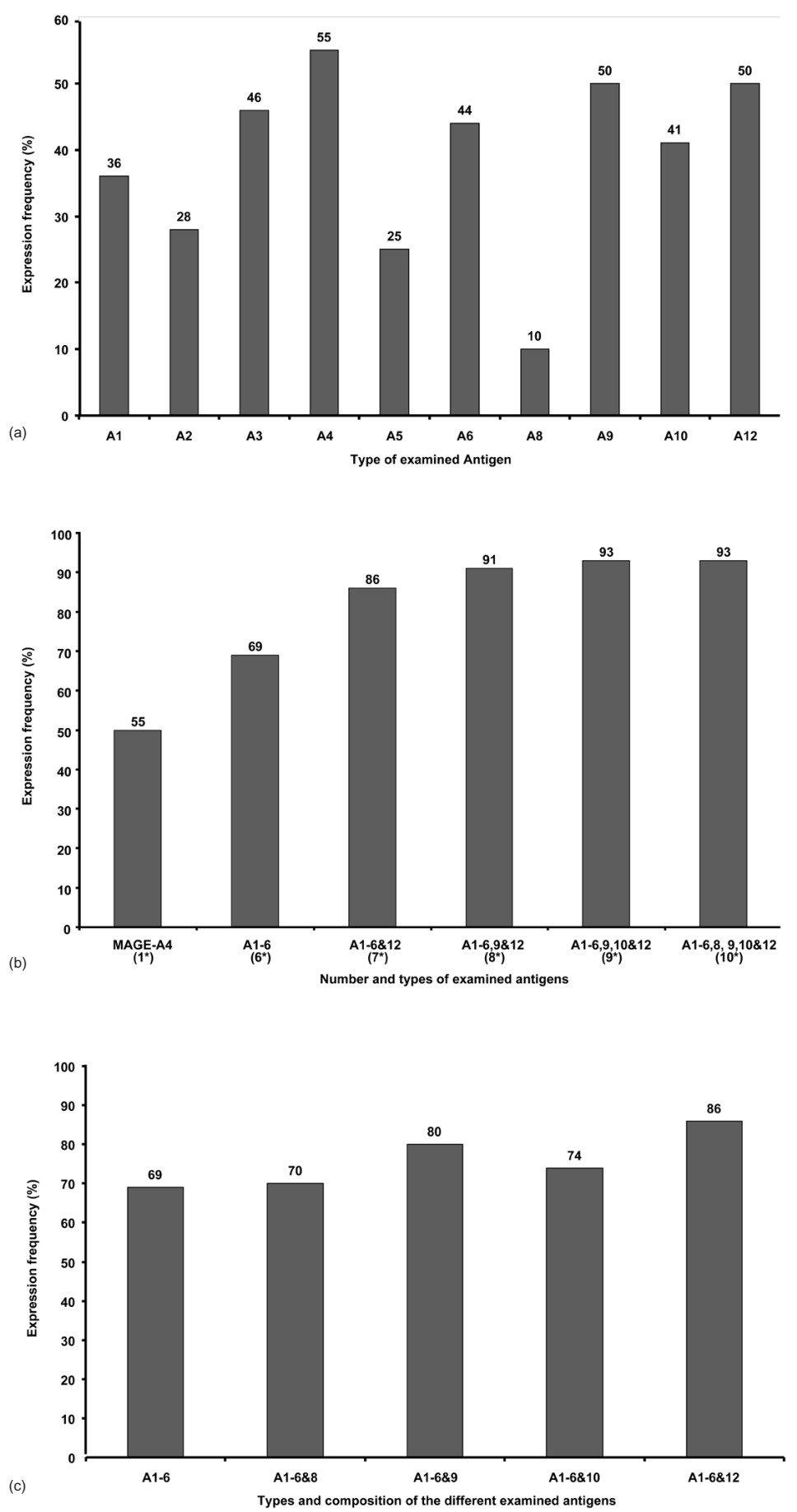

Fig. 2. Expression frequencies of single MAGE-A and at least one out of 10 investigated antigens in OSSC. (a) Expression frequencies of single antigen. (b) Expression frequency of at least one antigen which gradually increases by the addition of several antigens. (c-e) Increase of expression frequencies depending on the antigen taken into to account as a multiple marker system. (c) A highest frequency of expression when MAGE-A12 was applied as the 7th marker to the multiple marker system including MAGE-A1- A6 (86\%). (d) A highest frequency of expression when eight antigens which are MAGE-A1 to A6, MAGE-A9 and A12 were applied (91\%). (e) Highest number of frequency reached by the analysis of MAGE-A1 to A6, A9, A10 and A12 (93\%). When MAGE-A8 was added as the $10^{\text {th }}$ gene, no increase was found. 

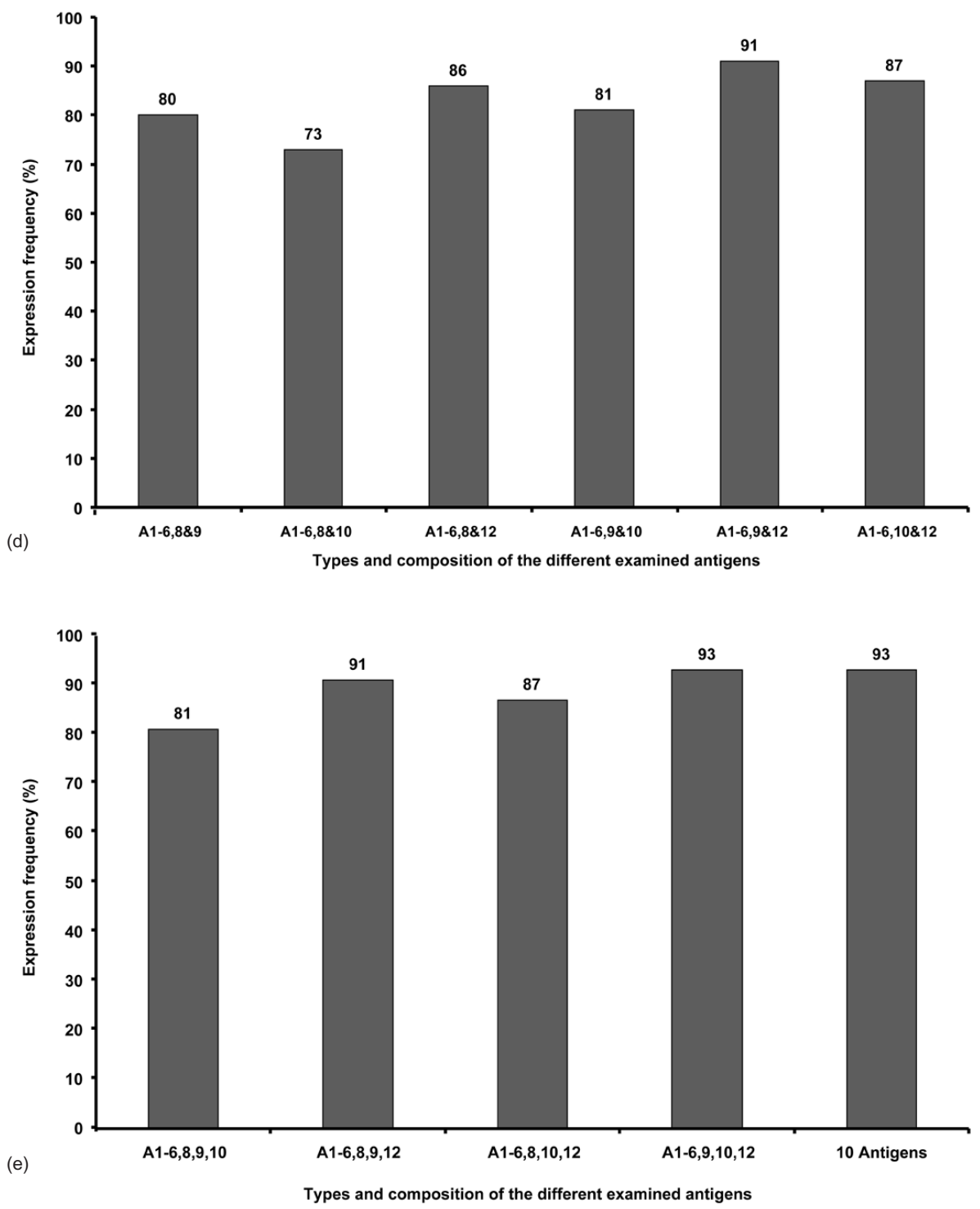

Fig. 2. continued.

at least one molecular marker. In the late clinical stages (Stage III and IV), the occurrence of frequency of at least one antigen was $100 \%$ (Table 2).

A constant increase of the expression frequencies was found by the inclusion of different MAGE-A to the analysis (Fig. 2). The expression frequency was $55 \%$ in maximum (MAGE-A4) when single antigen was applied (Fig. 2a, b). By simultaneous analysis of six MAGE-A (A1 to A6), the expression frequency increases to $69 \%$ (Fig. 2b). Depending upon the combination of the examined genes the expression frequency was elevated from $69 \%$ to $86 \%$ when seven genes were enrolled into the analysis. The highest in- crease of positive frequency (86\%) was found when MAGE-A12 was considered as a seventh candidate for the multiple markers. When MAGE-A8, A9 and A10 were considered as a 7 th, the frequency of a positive cases increased up to $70 \%, 80 \%$ and $74 \%$ respectively (Fig. 2c). However, when eight genes were enrolled into the analysis, the highest frequency was found by the MAGE-A1-6, A9 and A12 genes (91\%). With other configurations, the frequencies range between $73 \%$ and $87 \%$ (Fig. 2d). When nine genes were enrolled into the analysis, the highest frequency was found with the combination of MAGE-A1-6, A9, A10 and A12 (93\%) (Fig. 2e). The number of positive cases rose 
up to different levels by other antigen combinations. An exception was, when MAGE-A8 was included as the 9 th antigene to the combination of A1-A6, A9 and A10. In this case, expression frequency remains at $91 \%$ (Fig. 2d, e). Moreover, when MAGE-A8 was enrolled to the multiple marker expression analysis as the 10th candidate, no increase of expression frequency was detected (Fig. 2e). Hence, the highest number of positive cases displaying the highest sensitivity for tumor cell detection was found by multiple expression analysis of MAGE-A1-6, A9, A10 \& A12 (93\%).

\subsection{Association between MAGE-A mRNA detection and clinicopathological parameters}

The relationship between the different histological and clinicopathological parameters and the expression of al least one out of 10 MAGE-A was statistically evaluated. The results are summarized in Table 2. The expression of al least one antigen by the multiple expression analysis was strictly correlated to malignancy $(p<0.001)$. The expression frequency of at least one of the multiple markers applied was $100 \%$ when correlated to the late clinical stage (stage III and IV together) $(p=0.005)$. The expression frequencies were various and still significantly correlated when compared to stage I to IV separately ( $p=0.044)$. Moreover, there was no significance between expression of MAGE-A and tumor size $(p=0.87)$. However, the frequency of multiple MAGE-A expression was significantly associated to larger tumor size when tumor classification was grouped together as T1 \& T2 and T3 \& T4 $(p=0.024)$. In addition, no correlation was observed between gene expressions and state of lymph node when N0 was compared with $\mathrm{N} 1$ and N2 separately $(p>0.05)$. However, there was a statistical significance when N0 was compared with $\mathrm{N} 1+\mathrm{N} 2$ together $(\mathrm{N} 0<\mathrm{N} 1+\mathrm{N} 2)(p<0.05)$. It was also determined that all the tumor samples accompanied by the lymph node metastasis expressed at least one of the examined antigens. Additionally, there was a significant association between the multiple antigen expression and the histo-pathological differentiation degree in tumor cases $(p=0.031)$ (Table 2).

\section{Discussion}

In this study, expression of 10 MAGE-A was analyzed all together in number of OSSC samples in order to increase the sensitivity for the accuracy. According to previous studies, expression frequencies of the sin- gle subtypes were low and heterogeneous [16,23,24]. Thus, analysis of a single MAGE-A seems not to be quite sensitive for the diagnostic evaluation of OSCC. Elevated number of MAGE-A analysis does increase gradually the detection of sensitivity for OSCC cells when a certain combination was taken into account, especially when the amount of sample is not sufficient to perform analysis for each markers separately.

This study pointed out that MAGE-A1 to A6 should preferably be used for the detection of malignancy in OSCC, because these six genes can be detected concurrently even with a small amount of cells by the MAGEA1 to A6 assay $[15,16,23,24]$. It has also previously been determined that the expression frequency of MAGE-A can increase when MAGE-A12 is used as an additional 7th marker [24]. However, the main analysis can be gradually developed by using other antigens in order to reach the optimal sensitivity. In this study, the best result was determined by the analysis of seven genes when MAGE-A12 is included as the additional gene $(85 \%)$ [24]. Furthermore, the sensitivity was even higher when eight genes, A1-6, A9 and A12, were added in multiple marker combination $(91 \%)$. The highest sensitivity in the detection of malignancy was achieved by the analyses of following nine antigens which were MAGE-A1-A6, A9, A10 and A12 (93\%). Additional analysis of MAGE-A8 did not increase the sensitivity level. Therefore a multiple marker system which covers only nine antigenes, seems to be sufficient enough to reach the maximum sensitivity for the diagnosis of OSCC.

Expression of a single MAGE-A has already been detected in precursor lesions of intrahepatic cholangiocarcinoma and dysplastic precancerous tissues of lung cancer [32,33]. In addition, in the present study $81 \%$ of OSCC which were in early stage expressed at least one of the multiple antigens applied and it was claimed that analysis of multiple MAGE-A can also provide a high accurate and sensitive tool for the early diagnosis of OSCC. Moreover none of the NOM specimens expressed any MAGE-A indicating the high specificity to malignant cells. Thus, detection of multiple MAGE-A can also be applied as a predictor for the potential malignant transformation of oral pre-malignant lesions allowing the detection of already malignant transformed cells. Nevertheless, carefully designed studies are still needed to determine the expression profiles in mild dysplastic lesions of NOM that seem to have no potential of malignant transformation, and also in carcinoma in situ, , in order to clarify in particular the role of such molecular-biological methods as an additional tool for the cancer risk assessment of oral pre-malignancies. 
Furthermore, operated OSCC patients may still suffer from postoperative recurrence or metastases of neck lymph nodes which may indicate residual, occult malignant cells in tumor margins and also early DTCs in peripheral blood, bone marrow or lymph node as an intrinsic path [5]. Prognostic relevance of the tumor cell detection in hematopoietic cell compartments has already been studied in a variety of solid tumors [25, 34-38]. Expression of MAGE-A in the surgical margin and normal tissues adjacent to the oesophageal and lung cancer has been previously reported and was determined to be related to local recurrence. Thus, this may indicate that residual, occult OSCC cells may also be detected around tumor margins by the analysis of multiple expression profile of these genes and the occurrence of local recurrence may easily be predicted [33, 38]. Although, multiple expression analysis of MAGEA has already been applied to the early detection of lymph node metastasis and DTCs in blood and bone marrow of patients suffer from different solid tumors, carefully designed studies are still needed to determine the expression profiles of NOM at a distant and/or close to tumor margins to evaluate the role of MAGE-A [15, 23-25,28,39]. In addition, the present study determined a significant association between the expression frequency of multiple MAGE-A mRNA analysis and the positive lymph node metastases indicating the local recurrence irrespective of the tumor classification or size $(p=0.042)$. On the other hand in a previous study of Ferris et al. expression ratio of MAGE-A1, A2, A3, A4, A8, A10, A12 on Head and Neck SCC was investigated in tumor and benign lymph nodes in order to elucidate their role for molecular staging of the cervical lymph nodes. They found positive gene expression of MAGE-A, but the lowest or median detection level of single gene expression was at very low or at nondetectable level. Only MAGE-A3 showed detectable expression but display only a low tumor/highest benign node expression ratio and also a relatively low median expression in tumors. Thus this gene was omitted from the screening method, too [40]. However, it can be hypothesized that application of multiple MAGEA mRNA expression can also be used as an additional prognostic marker for the detection of even a very small or clinically not visible lymph node metastasis because they would express at least one of the multiple antigen applied in a high ratio. Nevertheless, expression pattern of MAGE-A in lymph node, bone marrow or peripheral blood sample of patients with OSCC still remains unclear, and warrants further investigations as well.
In conclusion, expression analysis of multiple MAGE-A is found to be more sensitive for the diagnostic evaluation of OSCC than the detection of a single MAGE-A. Based on the expression analysis of several MAGE-A, OSCC can be diagnosed early, surely with high probability. In addition, it also seems possible to detect residual, occult malignant cells in tumor margins and DTCs. Therefore, it is hypothesized that such a molecular-biological method may also represent an additional prognostic tool for the early diagnosis of OSCC and for the early prediction of recurrences/metastasis to provide a suitable individual therapy for each patient with OSCC.

\section{Acknowledgement}

Authors would like to indicate that this study has been supported by the grant of Johannes und Frieda Marohn-Stiftung, Erlangen and also would like to thank Ms. A. Krautheim-Zenk for the technical support.

\section{References}

[1] . D.M. Parkin, P. Pisani and J. Ferlay, Global cancer statistics, CA Cancer J Clin 49 (1999), 33-64.

[2] S.H. Landis, T. Murray, S. Bolden et al., Cancer statistics, 1999. CA Cancer J Clin 49 (1999), 8-31.

[3] D.M. Parkin, F. Bray, J. Ferlay et al., Global cancer statistics, 2002, CA Cancer J Clin 55 (2005), 74-108.

[4] K.D. Hunter, E.K. Parkinson and P.R. Harrison, Profiling early head and neck cancer, Nat Rev Cancer 5 (2005), 127-135.

[5] O. Bettendorf, J. Piffko and A. Bankfalvi, Prognostic and predictive factors in oral squamous cell cancer: important tools for planning individual therapy? Oral Oncol 40 (2004), $110-119$.

[6] D.P. Slaughter, H.W. Southwick and W. Smejkal, Field cancerization in oral stratified squamous epithelium; clinical implications of multicentric origin, Cancer 6 (1953), 963-968.

[7] T. Boon, E. De Plaen, C. Lurquin et al., Identification of tumour rejection antigens recognized by T lymphocytes, Cancer Surv 13 (1992), 23-37.

[8] P. van der Bruggen, C. Traversari, P. Chomez et al., A gene encoding an antigen recognized by cytolytic $\mathrm{T}$ lymphocytes on a human melanoma, Science 254 (1991), 1643-1647.

[9] E. De Plaen, K. Arden, C. Traversari et al., Structure, chromosomal localization, and expression of 12 genes of the MAGE family, Immunogenetics 40 (1994), 360-369.

[10] P. Chomez, O. De Backer, M. Bertrand et al., An overview of the MAGE gene family with the identification of all human members of the family, Cancer Res 61 (2001), 5544-5551.

[11] O. De Backer, K.C. Arden, M. Boretti et al., Characterization of the GAGE genes that are expressed in various human cancers and in normal testis, Cancer Res 59 (1999), 3157-3165.

[12] U.C. Rogner, K. Wilke, E. Steck et al., The melanoma antigen gene (MAGE) family is clustered in the chromosomal band Xq28, Genomics 29 (1995), 725-731. 
[13] A.A. Jungbluth, K.J. Busam, D. Kolb et al., Expression of MAGE-antigens in normal tissues and cancer, Int J Cancer $\mathbf{8 5}$ (2000), 460-465.

[14] A.A. Jungbluth, W.A. Silva, Jr., K. Iversen et al., Expression of cancer-testis (CT) antigens in placenta, Cancer Immun 7 (2007), 15.

[15] J.W. Park, T.K. Kwon, I.H. Kim et al., A new strategy for the diagnosis of MAGE-expressing cancers, $J$ Immunol Methods 266 (2002), 79-86

[16] M. Eura, K. Ogi, K. Chikamatsu et al., Expression of the MAGE gene family in human head-and-neck squamous-cell carcinomas, Int J Cancer 64 (1995), 304-308.

[17] M. Otte, M. Zafrakas, L. Riethdorf, et al., MAGE-A gene expression pattern in primary breast cancer, Cancer Res $\mathbf{6 1}$ (2001), 6682-6687.

[18] M.A. Kienstra, H.B. Neel, S.E. Strome, et al., Identification of NY-ESO-1, MAGE-1, and MAGE-3 in head and neck squamous cell carcinoma, Head Neck 25 (2003), 457-463.

19] P. Van Der Bruggen, Y. Zhang, P. Chaux et al., Tumor-specific shared antigenic peptides recognized by human T cells, Immunol Rev 188 (2002), 51-64.

[20] C.H. Chen, G.T. Huang, H.S. Lee, et al., High frequency of expression of MAGE genes in human hepatocellular carcinoma Liver 19 (1999), 110-114.

[21] K.D. Lee, H.H. Lee, H.B. Joo et al., Expression of MAGE A 1-6 mRNA in sputa of head and neck cancer patients - a preliminary report, Anticancer Res 26 (2006), 1513-1518.

[22] J. Li, Y. Yang, T. Fujie et al., Expression of the MAGE gene family in human gastric carcinoma, Anticancer Res 17 (1997), 3559-3563.

[23] J. Ries, S. Schultze-Mosgau, F. Neukam et al., Investigation of the expression of melanoma antigen-encoding genes (MAGEA1 to -A6) in oral squamous cell carcinomas to determine potential targets for gene-based cancer immunotherapy, Int $J$ Oncol 26 (2005), 817-824.

[24] J. Ries, E. Vairaktaris, N. Mollaoglu et al., Expression of melanoma-associated antigens in oral squamous cell carcinoma, J Oral Pathol Med 37 (2008), 88-93.

[25] P. Kufer, A. Zippelius, R. Lutterbuse et al., Heterogeneous expression of MAGE-A genes in occult disseminated tumor cells: A novel multimarker reverse transcription-polymerase chain reaction for diagnosis of micrometastatic disease, Cancer Res 62 (2002), 251-261.

[26] S. Kwon, S.H. Kang, J. Ro et al., The melanoma antigen gene as a surveillance marker for the detection of circulating tumor cells in patients with breast carcinoma, Cancer 104 (2005), 251-256.
27] I. Mecklenburg, D.F. Stratakis, R.M. Huber et al., Detection of melanoma antigen-A expression in sputum and bronchial lavage fluid of patients with lung cancer, Chest 125 (2004), $164 \mathrm{~S}-166 \mathrm{~S}$.

[28] I. Mecklenburg, D. Weckermann, A. Zippelius et al., A multimarker real-time RT-PCR for MAGE-A gene expression allows sensitive detection and quantification of the minimal systemic tumor load in patients with localized cancer, $J$ Immunol Methods 323 (2007), 180-193.

[29] L.H. Sobin and I.D. Fleming, TNM Classification of Malignant Tumors, 5th ed. (1997). Union Internationale Contre le Cancer and the American Joint Committee on Cancer, Cancer 80 (1997), 1803-1804.

[30] World Health Organization, International Histological Classification of Tumors, (2nd ed.), Berlin: Springer-Verlag, 1988.

[31] N. Mollaoglu, E. Vairaktaris, E. Nkenke et al., Expression of MAGE-A12 in oral squamous cell carcinoma, Dis Markers 24 (2008), 27-32.

[32] K. Tsuneyama, M. Sasaki, T. Shimonishi et al., Expression of MAGE-A3 in intrahepatic cholangiocarcinoma and its precursor lesions, Pathol Int 54 (2004), 181-186.

[33] S.J. Jang, J.C. Soria, L. Wang et al., Activation of melanoma antigen tumor antigens occurs early in lung carcinogenesis, Cancer Res 61 (2001), 7959-7963.

[34] S. Braun, F.D. Vogl, B. Naume et al., A pooled analysis of bone marrow micrometastasis in breast cancer, $N$ Engl J Med 353 (2005), 793-802.

[35] H.J. Gath and R.H. Brakenhoff, Minimal residual disease in head and neck cancer, Cancer Metastasis Rev 18 (1999), 109_ 126.

[36] K. Pantel and C. Alix-Panabieres, The clinical significance of circulating tumor cells, Nat Clin Pract Oncol 4 (2007), 62-63.

[37] K. Pantel and U. Woelfle, Micrometastasis in breast cancer and other solid tumors, J Biol Regul Homeost Agents 18 (2004), $120-125$.

[38] A. Zambon, S. Mandruzzato, A. Parenti et al., MAGE, BAGE, and GAGE gene expression in patients with esophageal squamous cell carcinoma and adenocarcinoma of the gastric cardia, Cancer 91 (2001), 1882-1888.

[39] B. Taback, A.D. Chan, C.T. Kuo et al., Detection of occult metastatic breast cancer cells in blood by a multimolecular marker assay: correlation with clinical stage of disease, Cancer Res 61 (2001), 8845-8850.

[40] R.L. Ferris, L. Xi, S. Raja el al., Molecular staging of cervical lymph nodes in squamous cell carcinoma of the head and neck, Cancer Res 65 (2005), 2147-2156. 


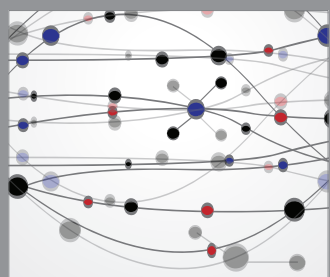

The Scientific World Journal
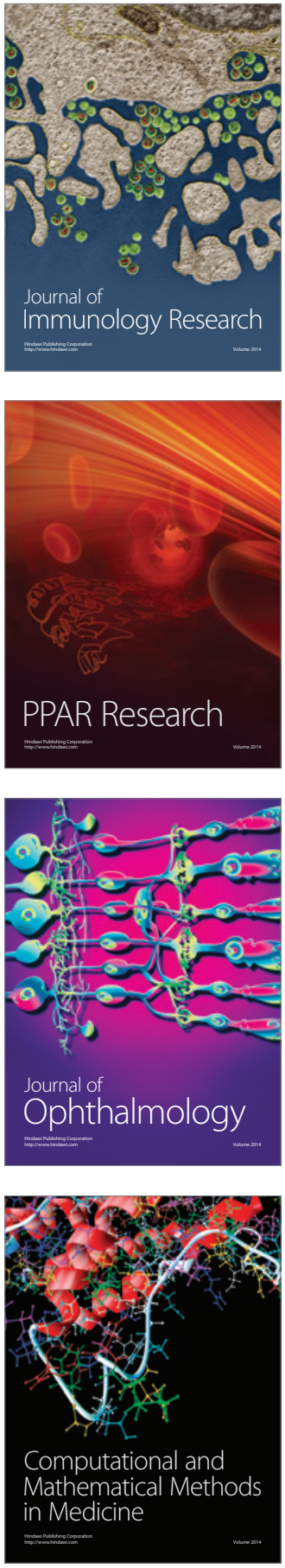

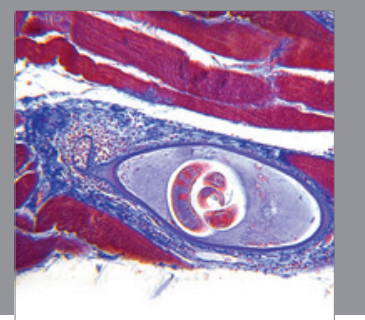

Gastroenterology

Research and Practice
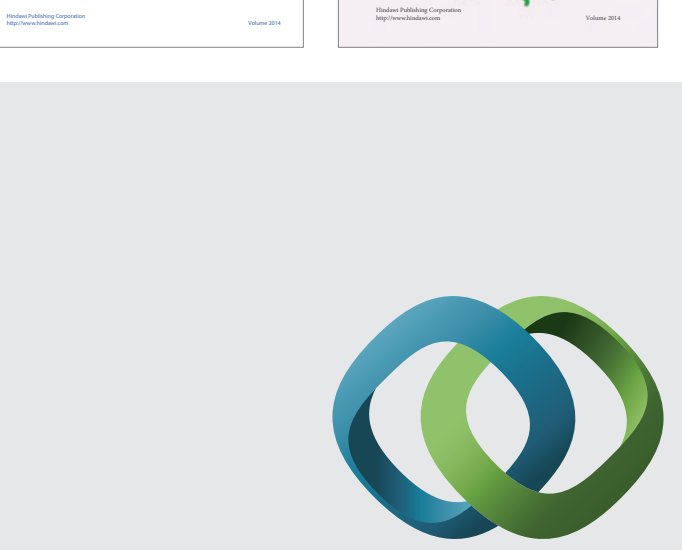

\section{Hindawi}

Submit your manuscripts at

http://www.hindawi.com
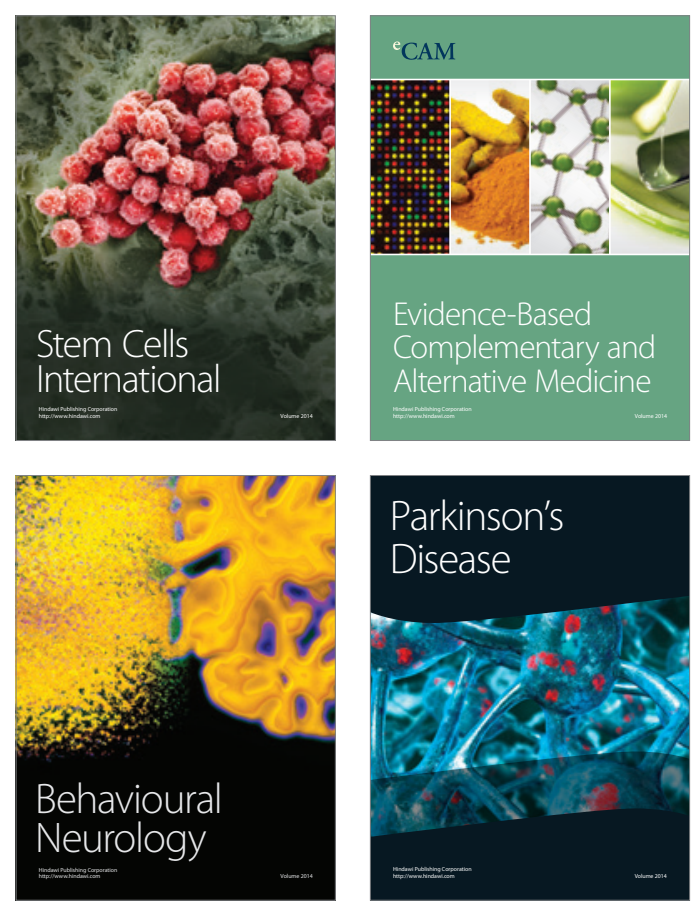

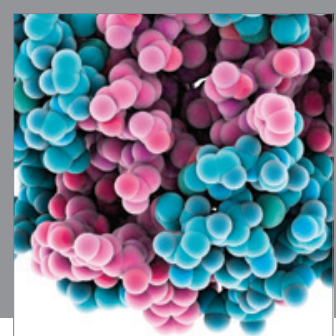

Journal of
Diabetes Research

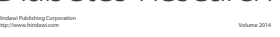

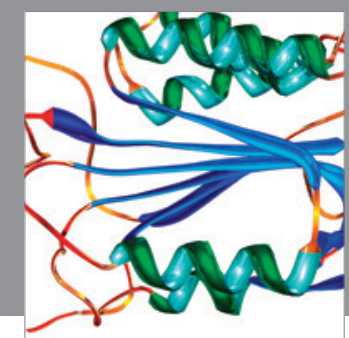

Disease Markers
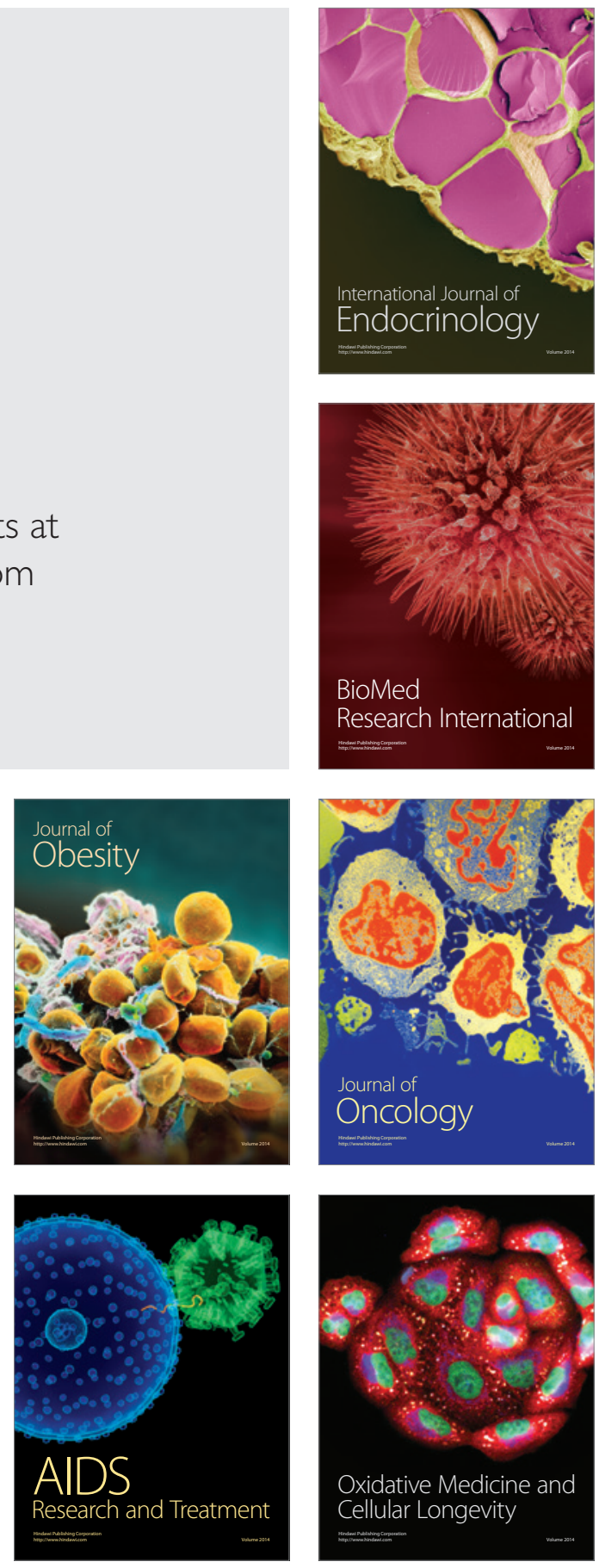